\title{
A Case Study on the Transformation from Proprietary to Open Source Software
}

\author{
Alma Oručević-Alagić and Martin Höst \\ Department of Computer Science, Lund University, Sweden \\ Alma.Orucevic-Alagic@cs.1th.se, Martin.Host@cs.lth.se
}

\begin{abstract}
This paper presents an extensive analysis of static software quality metrics changes for an open source enterprise database management system (DBMS), as the software was moved from the proprietary into open source software development environment. The software quality metrics of special interest for the research are cyclomatic complexity, effective lines of code, the degree of system modularity, and the amount of comments in the code.
\end{abstract}

\section{Introduction}

Popularization of OSS has influenced the conventional way in which companies perceive commercial value of software. The companies recognized that commercial value of product can come from other sources rather than conventional sale of software licenses. This research assesses the impact of source code changes made by OSS community to software that was transitioned from proprietary into open source, in terms of static software quality metrics. The case software analyzed is the Ingres database management system (DBMS) 2], which, according to many, has received a new breath of life after its release into the open source community.

\section{Background}

Stemlos 3] conducted code quality analysis in open source development for 100 applications written for Linux. It was determined that some open source products have lower quality of code produced in OSS environment then that which is expected as an industry standard.

The very roots of the case software reach back to the 1970s and UC Barkley, when the initial development of the software was started as open source. The same code base was modified and spawned into Sybase and Microsoft SQL server in 1980s. In 1994, the software was acquired by CA (Computer Associates) from the ASK Group, the company that created a proprietary version of the Ingres code. In order to increase the market share, $\mathrm{CA}$ decided to transform the product to open source in 2004, by implementing loss-leader/market positioner business model 44. In November of 2005, Computer Associates and Garnett and Helfirch capital created a new company, Ingres Corporation. The main role of Ingres 
Corporation is to oversee the open source development process. Today, Ingres customer base includes 10,000 enterprise customers, among which 136 belong to the Fortune 500 companies like 3M, Bea Systems, and Lufthansa 6].

The high level architecture of the case software is grouped into four major components:

Front End: Functionality covers user interface facilities.

Back End: Functionality covers DBMS server functionality.

Common: Functionality covers connectivity and communications between the front end the back end.

Utility: Functionality covers utility libraries that interact with operating system.

\section{Research Approach}

The following research questions were investigated during the research:

1. What parts of the Ingres DBMS software components went through the most source code changes in terms of source files added, changed, and deleted?

2. How did Ingres DBMS code base change under the OS community process in terms of static source code metrics?

It is important to highlight the objective of this study, i.e. to understand what changes that have been carried out, and not to assess or compare the case software to any other software. The study is conducted as a case study [5].

\subsection{Data Collection}

In order to analyze and compare code metrics of the most recent proprietary version, further referred as $2004 \mathrm{v}$, and open source version, further referred as $2008 \mathrm{v}$, of Ingres, the $2004 \mathrm{v}$ was obtained by directly contacting the Ingres Corporation. The 2008v was downloaded from the Ingres Open Source community web site in November of 2008.

A program that parses through the $2004 \mathrm{v}$ and $2008 \mathrm{v}$ code base was created, or more specifically, the files and subdirectories under the main src directory that contains all of the source files. The files were compared between the two code bases in order classify all files according to the following:

- File type 0 : Source files that can be found only in $2004 \mathrm{v}$

- File type 1 : Source files identical - unchanged between the 2004v and 2008v

- File type 2: Source files that were changed between the 2004v and 2008v

- File type 3: Source files that were added in 2008v

Metrics were measured in both versions are: lines of code $(L O C)$, effective lines of code $(E L O C)$, comment lines $(C)$, total cyclomatic complexity $(T C C)$, and file functions count $(F F C)$. All metrics are calculated on file level.

For coding purposes developers often use braces or parenthesis to make code more readable, but this practice can inflate $L O C$ metrics [7]. The ELOC metric 
takes into consideration all lines of code except blank only or comment only lines as well as the lines containing only standalone braces or parenthesis $(\{\},,()$, Thus, lines counted by the ELOC metric are a subset of the lines counted by the $L O C$ metric.

$C$ denotes the number of comment lines. The comment lines can appear by themselves on one physical line of code, or can be co-mingled.

The $T C C$ or total cyclomatic complexity metric, also known as McCabe's cyclomatic complexity, is the degree of logical branching per source file.

$F F C$, or total number of file functions, within a source file determines the modularity of the file. The $F F C$ metric combined with $E L O C$ metric produces average number of effective lines of code, $A E L O C=E L O C / F F C$. In the same way, the average cyclomatic complexity is calculated as $A C C=T C C / F F C$.

In addition to the above metrics, the amount of comments are of interest. Therefore a metric describing the relative number of comments in each file is calculated as $R C=C /(E L O C+C)$.

Metrics for each file were derived with a metrics tool and stored in a database together with file type information for analysis.

\subsection{Analysis}

Analysis with respect to research question 1 was conducted by determining the percentage changes in terms of file type 0 , file type 1 , file type 2 , and file type 3 per major components of the source code.

When analysing research question 2, the differences between the different versions of the case software were investigated with hypothesis tests. The null hypotheses state that the code changes made to $2004 \mathrm{v}$, resulting in $2008 \mathrm{v}$, had no impact on code metrics.

Let $T=\{0,1,2,3\}$ denote file types according to above and let $M=\{A E L O C$, $A C C, R C\}$ denote the different metrics of interest, so that $\mu_{m}\left(v, T_{s}\right)$ represents the expected mean of metric $m \in M$ for all files of types $T_{s} \subseteq T$ in version $v$. Then the following null hypotheses have been defined:

$$
\begin{aligned}
H 0_{m, \text { changed }} & : \mu_{m}(2004 v,\{2\})=\mu_{m}(2008 v,\{2\}) \\
H 0_{m, \text { new }} & : \mu_{m}(2004 v,\{0,1,2\})=\mu_{m}(2008 v,\{3\}) \\
H 0_{m, \text { all }} & : \mu_{m}(2004 v,\{0,1,2\})=\mu_{m}(2008 v,\{1,2,3\})
\end{aligned}
$$

That is, three null hypotheses have been formulated for each metric in $M$ so that there is one concerning only the changed files $\left(H 0_{m, c h a n g e d}\right)$, one concerning all files from $2004 \mathrm{v}$ and only newly added files to $2008 \mathrm{v}\left(H 0_{m, n e w}\right)$, and one concerning all the files in $2004 \mathrm{v}$ and $2008 \mathrm{v}\left(H 0_{m, \text { all }}\right)$. This means that $|M| \times 3=$ $3 \times 3=9$ null hypotheses and equally many alternative hypotheses have been defined in total.

Analysis of data for distributions of metrics results for version $2004 \mathrm{v}$ and $2008 \mathrm{v}$ were performed and it was determined that data for the metrics do not follow normal distribution. Hence in order to compare distribution of the metrics, non parametric tests, Mann-Whitney and Wilcoxon were performed. The Wilcoxon 
Signed-Rank Test for matched pairs was used in order to compare paired data sets (i.e., in analysis of $H 0_{m \text {,changed }}$ ), and the Mann-Whitney U test was used to compare un-paired data (i.e., in analysis of $H 0_{m, a l l}$ and $H 0_{m, n e w}$ ).

\section{Results}

Not all of the source code subdirectories will be analyzed in more detail, but only front, back, common, gl, and cl, since these directories contain almost $95 \%$ of the code. Hence, the most of the source files are located under/src/front directory or $54.7 \%$ of all $2008 \mathrm{v}$. In the second place is src/cl directory housing $15.40 \%$ of source files in $2008 \mathrm{v}$, followed by the src/back and src/common, housing $14.06 \%$ and $10.44 \%$ of all $2008 \mathrm{v}$ source files, respectively. Thus, these four directories contain $94.6 \%$ of $2008 \mathrm{v}$ source files.

Under the src/front directory the components that belong to the front end layer of the software are stored. Over $50 \%$ of all changes in the front end layer are due to the addition of the new source file components (type 3). Another $33 \%$ of changes are due to changes (file type 2). Thus, around $88 \%$ of front end source files have been changed since the case software went open source. Under src/cl library source files for Ingres Compatibility Library are housed. This library grew $69 \%$ between $2004 \mathrm{v}$ and $2008 \mathrm{v}$, that is it contains $69 \%$ of file type 3 files. The src/back end components are deemed very important as the proper functioning of these components significantly affects database performance. The back end components went through the least amount of source code changes and additions, having $67.5 \%$ of code unchanged (file type 1) between the $2004 \mathrm{v}$ and $2008 \mathrm{v}$. It also contains the least number of file additions (file type 3 ), thus only having $2.92 \%$ of the total number of the source files added (file type 3 ). Finally the src/common contains components used by both, front and back end. The common components contain $49.05 \%$ of file type 1 , or almost half of its components are same for $2004 \mathrm{v}$ and $2008 \mathrm{v}$. It can be observed that $19.5 \%$ of its file were of file type 3 , or newly added components.

Table 1 displays code metric statistics summarized for the entire source code base of $2004 \mathrm{v}$ and $2008 \mathrm{v}$. Hence, it can be observed that the number of file functions, lines of code and effective lines of code has increased. As one would expect, the higher number of functions and lines of code produced higher values for total cyclomatic complexity of $2008 \mathrm{v}$ code compared to $2004 \mathrm{v}$.

The results of hypothesis testing for the stated hypotheses are presented in Table 2 (significance level 0.05).

Concerning $A E L O C$, this metric is somewhat increased for changed files, meaning that when files are changed the functions in the files have become somewhat larger. For new files the metric is much lower than for old files, meaning that functions in new files are smaller than in older files. In total, looking at all files, the metric is higher in the new version than in the older version. The differences are statistically different for changed code and new code compared to old code, but not for all code. 
Table 1. Summary of source code metrics for the whole system

\begin{tabular}{|l|l|l|}
\hline Code Metric & $2004 \mathrm{v}$ & $2008 \mathrm{v}$ \\
\hline \hline Total $L O C$ & 840,502 & $1,442,225$ \\
\hline Total $E$ LOC & 650,055 & $1,110,261$ \\
\hline Total $C$ & 484,349 & 630,635 \\
\hline Total TCC & 167,753 & 300,493 \\
\hline Total FFC & 15,588 & 45,216 \\
\hline
\end{tabular}

Table 2. Mean values and results of hypothesis tests

\begin{tabular}{|l|l|l|l|l|}
\hline$H 0$ & mean 2004 & mean 2008 & $p$ & reject $H 0$ \\
\hline \hline$H 0_{A E L O C, \text { changed }}$ & 41.35 & 41.69 & $<0.001$ & yes \\
\hline$H 0_{A C C, \text { changed }}$ & 10.47 & 10.80 & $<0.001$ & yes \\
\hline$H 0_{R C, \text { changed }}$ & 0.53 & 0.54 & 1 & no \\
\hline$H 0_{A E L O C, \text { new }}$ & 23.68 & 11.85 & 0.0042 & yes \\
\hline$H 0_{A C C, \text { new }}$ & 6.12 & 2.80 & 0.01 & yes \\
\hline$H 0_{R C, \text { new }}$ & 0.56 & 0.42 & $<0.001$ & yes \\
\hline$H 0_{A E L O C, \text { all }}$ & 23.68 & 19.02 & 0.1383 & no \\
\hline$H 0_{A C C, \text { all }}$ & 6.12 & 4.85 & 0.1841 & no \\
\hline$H 0_{R C, \text { all }}$ & 0.56 & 0.50 & $<0.001$ & yes \\
\hline
\end{tabular}

Concerning $A C C$ the same type of observation as for $A E L O C$ can be made. For changed files the complexity is slightly higher and for new files the complexity is much lower. For $R C$ there is no significant difference for changed code, but for new code there are significantly less comments. In total there is relatively less comments in the new version compared to the old version.

\section{Conclusions}

The conducted analysis have shown that over half of the changes made to the case source code were made in the front end group of source code components, while the least of the changes were seen in the back end components. There can be many reasons for this, e.g. simply that more changes were needed in these components, but another reason may be that these are nearer to the interest of the new community that was formed during the open source transition process.

The results of comparison of code quality metrics between all files in $2004 \mathrm{v}$ and new files in 2008v show significant and large decrease in $A C C$ and $A E L O C$, that is, significant and large increase in quality metrics for code developed by the OSS community. The code quality decrease in metrics smaller than the increase of the changed files, and as a result the code quality metrics for $2008 \mathrm{v}$ are higher than those of the $2004 \mathrm{v}$, but this increase in code quality is not significant. This means that the overall code quality metrics, in terms of average cyclomatic complexity and the average effective lines of code per function has increased somewhat for changed code, and decreased rather much for new code. This can 
be interpreted as an improvement for added code. The number of comment lines per effective lines of code $A C C$ has decrased and there are significantly less comments in newly added code. At the same time the number of comments per effective lines of code $(R C)$ has seen significant decrease between the $2004 \mathrm{v}$ and $2008 \mathrm{v}$ of source code base. Hence, while there was a small improvement in $A C C$ and $A E L O C$, the lower number of comments per effective lines of code suggests that code in OSS community was not documented as much as in closed source environment.

The transition of the software was also accompanied by $100 \%$ increase in customer base, out of which some 138 customers belong to the Fortune 500 group, and $32 \%$ revenue increase reported for the 2008 .

For companies planning to go open source, this study can provide an example on how the OSS community can impact static software quality metrics.

\section{Acknowledgments}

The authors would like to express their gratitude to the Ingres Corporation for providing us with a last proprietary version of the software. This work was partly funded by the Industrial Excellence Center EASE - Embedded Applications Software Engineering, (http://ease.cs.lth.se).

\section{References}

1. Fenton, N.E., Pfleeger, S.L.: Software Metrics: A Rigorous and Practical Approach, Revised. PWS Publishing Company, ITP International Thomson Publishing Company (1998)

2. IngresWebSite. Official web site of ingres corporation (2009), http://ingres.com/

3. Oikonomou, A., Stamelos, I., Angelis, L.: Code quality analysis in open source development. Information Systems Journal 12(1), 43-60 (2002)

4. Raymond, E.S.: The Cathedral and the Baazar. O'Reilly Media, Inc., Sebastopol (2001)

5. Runeson, P., Höst, M.: Guidelines for conducting and reporting case study research in software engineering. Empirical Software Engineering 14, 131-164 (2008)

6. Assay, M.: Web server survey (February 2009), http://news.cnet.com/8301-13505_3-10156188-16.html

7. RSM Effective lines of code eloc metrics for popular open source software linux kernel 2.6.17, firefox, apache hppd, mysql, php using rsm (2008), http://msquaredtechnologies.com/m2rsm/docs/rsm_metrics_narration.htm 